\title{
Syntheses and Anticancer Activities of Novel Glucosylated (-)-Epigallocatechin-3-Gallate Derivatives Linked via Triazole Rings
}

\section{Cheng-Ting Zi}

Yunnan Agricultural University https://orcid.org/0000-0002-2367-3617

Bo-Ya Shi ( $\square$ shiboya7@163.com)

Yunnan Agricultural University

\section{Ze-Hao Wang}

Yunnan Agricultural University

Ning Zhang

Yunnan Agricultural University

Yin-Rong Xie

Yunnan Agricultural University

Xiu-Li Sun

Yunnan Agricultural University

Hao-Nan Yang

Yunnan Agricultural University

Yi-Long Wu

Yunnan Agricultural University

Xuan-Jun Wang

Yunnan Agricultural University

Jun Sheng

Yunnan Agricultural University

\section{Original Article}

Keywords: EGCG, butyrylated glucosides, antitumor activity, CuAAC, synthesis

Posted Date: February 11th, 2021

DOI: https://doi.org/10.21203/rs.3.rs-182372/v1

License: (c) (1) This work is licensed under a Creative Commons Attribution 4.0 International License.

Read Full License 


\section{Abstract}

Novel glucosylated (-)-epigallocatechin-3-gallate derivatives 10 - 13 having the EGCG analogues conjugated to the D-glucosyl azide were synthesized by carrying out the copper-catalyzed azide-alkyne cycloaddition (CuAAC) reaction, and were evaluated for their cytotoxicities against a panel of five human cancer cell lines (HL-60, SMMC-7721, A-549, MCF-7 and SW480) using MTT assays. Compounds 10 and 11 showed the highest levels of cytotoxicity against the $\mathrm{HL}-60$ cells with $\mathrm{IC}_{50}$ values of $4.57 \mu \mathrm{M}$ and 3.78 $\mu \mathrm{M}$, respectively, and showed moderate selectivity towards cancer cell lines. Compound $\mathbf{1 1}$ was also shown to induce apoptosis in $\mathrm{HL}-60$ cells. Most notably, inclusion of the perbutyrylated glucose residue in an EGCG derivative was concluded to lead to increased anticancer activity.

\section{Introduction}

Tea (Camellia sinensis (Linnaeus) O. Kuntze) was first taxonomically described in 1753 by Carl Linnaeus in SpeciesPlantarum. Two species of tea have been identified, namely black tea (Thea bohea) and green tea (Thea viridis) [1]. Four varieties of Camellia sinensis have been identified, including Camellia sinensis var. assamica, Camellia sinensis var. sinensis, Camellia sinensis var. dehungensis, and Camellia sinensis var. pubilimba, which are mainly distributed in the understory of forests of broad-leaved evergreen trees at altitudes of between 100-2,200 m [2]. The Chinese have been using tea as a drink since $3000 \mathrm{BC}$, and the subspecies: var. sinensis (China tea) and var. assamica (Assam tea) are found in China.

Green tea is one of the drinks most widely consumed by people around the world, perhaps second most after water, due to its health, sensory, stimulant, relaxing and cultural properties [3]. Catechins are the primary compounds responsible for the claimed health benefits of green tea, including its antioxidant and anti-inflammatory properties. The major catechins in green tea including (-)-epicatechin (EC, 1), (-)epicatechin-3-gallate (ECG, 2), (-)-epigallocatechin (EGC, 3) and (-)-epigallocatechin-3-gallate (EGCG, 4) (Figure 1) have been reported to display numerous biological activities [4-6]. EGCG is the most abundant catechin found in green tea and has been reported to display physiological activities stronger than those of the other catechins [7-9] and to display many types of biological activities including anti-oxidative, anti-inflammatory, anti-cancer, anti-infection and neuroprotective activities [10-12].

However, the use of EGCG is often hindered by problems such as being easily oxidized, readily degraded in aqueous solution and poorly intestinal absorbed in the intestines $[13,14]$. To obtain more potent analogues and overcome this problem of poor intestinal absorption, many semisynthetic derivatives such as permethyl EGCG [15], peracetyl EGCG [16], EGCG monoester derivatives [17], and EGCG glycosides [13, 18-21] have been developed. In recent years, the use of glycoconjugates of small-molecule anticancer drugs has become an attractive strategy for improving drug efficacy [22, 23]. In our previous study, we reported the syntheses and cytotoxicities of glucosylated EGCG derivatives, we found that, in aqueous solution, EGCG glucosides displayed higher activities against cells of human breast cancer cell lines and higher levels of stability than did EGCG [21]. 
Due to the ability of terminal alkyne and an azides to undergo copper-catalyzed [ $3+2$ ]-cycloadditions with azides to generate substituted triazole rings [24] and due to butyrate having been shown to be a histone deacetylase (HDAC) inhibitor and to display anticancer effects with promising therapeutic potential [25], we set out in the current work to chemically synthesized glucosylated (-)-epigallocatechin-3gallate derivatives linked via triazole rings and to characterize their in vitro anticancer activities against five human cancer cell lines, including HL-60 (leukemia), SMMC-7721 (hepatoma), A-549 (lung cancer), MCF-7 (breast cancer) and SW480 (colon cancer). In addition, chemical informatics analyses of these compounds were carried out, and the chemical properties of the compounds were correlated with their anticancer activity.

\section{Results And Discussion}

\section{Chemistry}

The novel triazole-linked glucose-(-)-epigallocatechin-3-gallate derivatives 10 - 13 were synthesized according to the synthetic route shown in Scheme 1. Here, 2,3,4,6-tetra-0-butyryl-1-azido-a-Dglucopyranose 7 was readily prepared from D-glucose by using a method similar to that reported in the literature [26]. And O-alkylated EGCG analogues 8 and $\mathbf{9}$ were prepared in 30\%-45\% yields by reacting EGCG (4) with potassium carbonate $\left(\mathrm{K}_{2} \mathrm{CO}_{3}\right)$ and propargyl bromide at room temperature for $12 \mathrm{~h}$. Then, compound $\mathbf{7}$ was allowed to react with EGCG analogues $\mathbf{8}$ and $\mathbf{9}$ at their terminal alkynes in the presence of copper (II) acetate and sodium ascorbate in $t-\mathrm{BuOH}-\mathrm{H}_{2} \mathrm{O}(1: 1)$ as the solvent for $2 \mathrm{~h}$ to yield $2,3,4,6-$ tetra-0-butyryl-a-D-glucopyranosyloxy-1,2,3-triazol-1-yl-(-)-epigallocatechin-3-gallates 10 and 11 in 77$80 \%$ yields $[24,27]$. Finally, compounds 10 and 11 were treated with potassium hydroxide $(\mathrm{KOH})$ solution (dissolved in $\mathrm{CH}_{3} \mathrm{OH}$ ) in $\mathrm{CH}_{3} \mathrm{OH}$ at $0{ }^{\circ} \mathrm{C}$ for $72 \mathrm{~h}$ to yield triazole-linked glucose-EGCG derivatives 12 and 13 with $52 \%$ to $55 \%$ yields [28].

The structures of all of the synthesized compounds were characterized using ${ }^{1} \mathrm{H}$-NMR spectroscopy, ${ }^{13} \mathrm{C}$ NMR spectroscopy, electrospray ionization mass spectrometry (ESI-MS), and high-resolution mass spectrometry (HRESI-MS). ESI-MS and HRESI-MS of all compounds showed the $[\mathrm{M}+\mathrm{Na}]^{+} /\left[\mathrm{M}+\mathrm{H}^{+} /[\mathrm{M}-\right.$

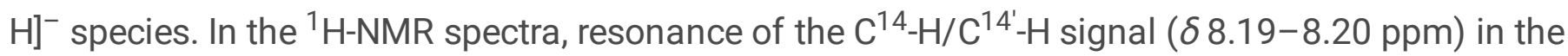
aromatic region confirmed the formation of the triazole ring. The structures were further confirmed upon analysis of their ${ }^{13} \mathrm{C}$-NMR spectra, which showed the two characteristic carbon signals at $145.4-146.7$ $\operatorname{ppm}\left(\delta_{\mathrm{C}-14} / \delta_{\mathrm{C}-14^{\prime}}\right)$ and the others at $124.7-126.9 \mathrm{ppm}\left(\delta_{\mathrm{C}-13} / \delta_{\mathrm{C}-13^{\prime}}\right)$, corresponding to the triazole residue. The synthesized compounds 12 and 13 were further analyzed using 2D-NMR spectroscopy (Fig. 2); the heteronuclear multiple bond correlation $(\mathrm{HMBC})$ of compound 12 showed a strong correlation between $\mathrm{C}^{12}-\mathrm{H}(5.11 \mathrm{ppm})$ and C-4" (139.4 ppm) of the D ring. This signal indicated an attachment of the triazolelinked ring to the C-4" of EGCG. Similarly, the HMBC of compound $\mathbf{1 3}$ indicated the occurrence of coupling between $\mathrm{C}^{12}-\mathrm{H}(5.11 \mathrm{ppm})$ and $\mathrm{C}-4 "(139.4 \mathrm{ppm})$ of the $\mathrm{D}$ ring and also between $\mathrm{C}^{12}-\mathrm{H}(5.21 \mathrm{ppm})$ and C$4^{\prime}(136.3 \mathrm{ppm})$ of the $B$ ring. These results indicated that the attachment of two triazole-linked ring one to the $\mathrm{C}-4$ ' and $\mathrm{C}-4$ " position of EGCG. The chemical shift for the proton at $\mathrm{C}^{4}-\mathrm{H}$ was observed to the coupled 
with $\mathrm{C}-2$ and $\mathrm{C}-3$, those for $\mathrm{C}^{2}-\mathrm{H}$ and $\mathrm{C}^{6}-\mathrm{H}$ occurred with $\mathrm{C}-2$ and $\mathrm{C}^{21}-\mathrm{H}$, that for $\mathrm{C}^{6 "}-\mathrm{H}$ occurred with $\mathrm{C}-11$, and that for $\mathrm{C}^{2}-\mathrm{H}$ occurred with $\mathrm{C}-11$ in both compounds 12 and 13.

\section{In vitro anti-proliferative activity}

The triazole-linked glucose-(-)-epigallocatechin-3-gallate derivatives 10 - 13 were evaluated for their cytotoxicities against five human cancer cell lines, including HL-60, SMMC-7721, A-549, MCF-7 and SW480. The compounds EGCG and cisplatin were used as positive controls. The screening procedure was based on the standard MTT method [26]. Their activities were expressed as $\mathrm{IC}_{50}$ values (concentration of drug inhibiting $50 \%$ cell growth) and the data are presented in Table 1.

The compounds having a free glucose residue namely compounds 12 and 13 show weaked activity levels $\left(\mathrm{IC}_{50}>40 \mu \mathrm{M}\right)$ toward cells of the three cancer cell lines SMMC-7721, A-549 and SW480. In contrast, the derivatives containing each a each a perbutyrylated glucose residue, namely compounds 10 and 11 showed higher activity levels, and they showed the highest cytotoxicity levels agaisnt HL-60 cells, with $\mathrm{IC}_{50}$ values of $4.57 \mu \mathrm{M}$ and $3.78 \mu \mathrm{M}$, respectively; they were also found to more potent than the control drug EGCG, which displayed $I_{50}>40 \mu \mathrm{M}$ against each of the five cancer cell lines. Interestingly, all of the EGCG derivatives showed good levels of cytotoxicity against MCF cells with $\mathrm{IC}_{50}$ values in the range 28.24-39.89 $\mu \mathrm{M}$. Based on these results taken together, we concluded that perbutyrylation of the glucose residue of the EGCG scaffold lead to increased anticancer activity.

\section{Selectivities of the compounds}

To evaluate the degrees of selectivity of the most cytotoxic compounds, namedly 11 and 12, their growth inhibitory effects on cells of a normal human bronchial epithelial cell line (BEAS-2B) were measured (Table 1). The selectivity index (SI) values of compounds 11, 12 and cisplatin are presented in Table 2. Compounds 11 and 12 showed moderate selectivity toward cancer cell lines with SI values in the range of 1.0-8.4 for all cells tested.

\section{Induction of cell apoptosis}

Given that the EGCG derivative 11 exhibited significant inhibitory activity of cancerous cell growth in HL60 cells, we studied further the ability of compound $\mathbf{1 1}$ to induce cell death through apoptosis. To carry out this study, the tested HL-60 cells were stained with annexin V, and compound $\mathbf{1 1}$ was administered at a concentration of $8 \mu \mathrm{M}$. Significantly higher amounts of compound $\mathbf{1 1}$ were detected in HL-60 cells undergoing apoptosis than in the untreated control (Fig. 3A-B). We also determined the expression levels of caspase-3 and PARP, which are the hallmarks of apoptosis and play crucial roles in the cellular process. For this purpose, samples of HL-60 cells were treated with compound 11, respectively, concentrations of 2, 4 and $8 \mu \mathrm{M}$ for $12 \mathrm{~h}$ and the expression levels of caspase-3, PARP, cleaved-caspase-3 and cleaved-PARP were monitored using western blot analysis. The treatment of HL-60 cells with compound 11 was found to be associated with increased levels of expression of cleaved-caspase-3 and cleaved-PARP in a dose-dependent manner (Fig. 3C). Compared to the untreated control, compound 11 
apparently induced a significant increase in the expressed levels cleaved-caspase-3 and cleaved-PARP and a decrease in those of caspase-3 and PARP.

\section{Physicochemical property}

As compounds 12 and 13 showed each weak activity in vitro, we calculated the Clogp values of $10-13$ by MarvinSketch version 5.3.8 [29], and the data are shown in Table 3. ClogP values of only 0.50 and 2.08 were calculated for, respectively, compounds 12 and 13. But higher ClogP values of 6.84 and 10.61 were calculated for, respectively, compounds 10 and 11 . Based on these results, compounds 12 and 13 were expected to display lower of cell permeability than were compounds $\mathbf{1 0}$ and $\mathbf{1 1}$.

\section{Solubility}

We also determined the water solubilities of the EGCG and EGCG derivatives (10-13), and these results are shown in Table 4. The solubility of EGCG was measured to be $16.40 \mathrm{mM}$, whereas those of compounds 10-13 were measured to be $5.71 \mathrm{mM}, 1.05 \mathrm{mM}, 281.17 \mathrm{mM}$ and $512.88 \mathrm{mM}$, respectively. That is compounds 12 and 13 (those each having a free glucose residue) showed, respectively, 17 and 31 times higher levels of solubility in water than did EGCG; while compounds $\mathbf{1 0}$ and $\mathbf{1 1}$ (the derivatives each containing a perbutyrylated glucose residue showed respectively, solubility levels only fractions ( 0.3 and 0.06) of that of EGCG. Taken together, these data indicated a trend of higher aqueous solubility levels for those compounds containing more glycol units.

\section{Conclusion}

In summary, a series of novel glucosylated (-)-epigallocatechin-3-gallate derivatives have been synthesized by carrying out the copper-catalyzed azide-alkyne cycloaddition (CUAAC) reaction. According to the results of in vitro proliferation inhibitory activity against a panel of five human cancer cells (HL-60, SMMC-7721, A-549, MCF-7 and SW480), Compounds 10 and 11 showed the highest levels of cytotoxicity against the $\mathrm{HL}-60$ cells with $\mathrm{IC}_{50}$ values of $4.57 \mu \mathrm{M}$ and $3.78 \mu \mathrm{M}$, respectively. Compounds with perbutyrylated glucose residue was concluded to lead to increased anticancer activity. Further research for modifying and enhancing their biological activity potential is undergoing based on the present data.

\section{Experimental}

\section{Materials and measurements}

D-glucose and $n$-butyric anhydride were purchased from Aladdin Chemical Co., Ltd (Guangzhou, China); (-)-epigallocatechin-3-gallate was obtained from Chengdu Proifa Technology Development Co., Ltd (Chengdu, China); and 3-(4,5-dimethyl-thiazol-2-yl)-2,5-diphenyltetrazolium bromide (MTT) was purchased from Sigma-Aldrich (St. Louis, MO, USA). All reagents were commercially available and used without further purification unless indicated otherwise. The melting points were measured by using an X-4 melting point apparatus and were uncorrected. Optical rotations data were obtained using a Jasco P- 
1020 Automatic Digital Polariscope. MS data were obtained in ESI mode using an API Qstar Pulsar instrument; HRMS data were obtained in ESI mode using LCMS-IT-TOF apparatus (Shimadzu, Kyoto, Japan); ${ }^{1} \mathrm{H}-\mathrm{NMR}$ and ${ }^{13} \mathrm{C}$-NMR spectra were recorded using a Bruker DRX-500 instrument (Bruker BioSpin $\mathrm{GmbH}$, Rheinstetten, Germany) with tetramethylsilane (TMS) as an internal standard. Column chromatography (CC) was performed with a silica gel (200 - 300 mesh; Qingdao Makall Group CO., LTD; Qingdao; China). All reactions were monitored using thin-layer chromatography (TLC) on silica gel plates, which were visualized using ultraviolet light $(254 \mathrm{~nm})$ and/or $10 \%$ phosphomolybdic acid/EtOH. All cancer cell lines (HL-60, SMMC-7721, A-549, MCF-7, and SW480) were obtained from a Shanghai cell bank in China.

\section{Synthesis of 2,3,4,6-tetra-0-butyryl-1-azido-a-D-glucopyranose (7)}

D-glucose $(1.8 \mathrm{~g}, 10 \mathrm{mmol})$ was suspended in $n$-butyric anhydride $(3.5 \mathrm{~mL}, 20 \mathrm{mmol})$ and stirred at $0{ }^{\circ} \mathrm{C}$. lodine $(100 \mathrm{mg})$ was added to this stirred suspension and the stirring was continued for $1 \mathrm{~h}$. The resulting reaction mixture was diluted with $\mathrm{CH}_{2} \mathrm{Cl}_{2}(50 \mathrm{~mL})$ and washed successively with aqueous saturated $\mathrm{Na}_{2} \mathrm{~S}_{2} \mathrm{O}_{3}$ and aqueous saturated $\mathrm{NaHCO}_{3}$. The resulting organic layer was then dried over $\mathrm{Na}_{2} \mathrm{SO}_{4}$ and concentrated under vacuo to give the crude per-butyrylated product $(5.2 \mathrm{~g}, 99 \%)$. This crude product (5.2 $\mathrm{g}, 10 \mathrm{mmol})$ was dissolved in $\mathrm{CH}_{2} \mathrm{Cl}_{2}(30 \mathrm{~mL})$, and hydrobromic acid $(5 \mathrm{~mL})$ was slowly added dropwise to this solution while the resulting mixture was slowly and stirred at $0{ }^{\circ} \mathrm{C}$. The mixture was stirred for $8 \mathrm{~h}$ until no starting material was detected using the TLC analysis. The reaction mixture was diluted with $\mathrm{CH}_{2} \mathrm{Cl}_{2}(30 \mathrm{~mL})$ and washed with aqueous saturated $\mathrm{NaHCO}_{3}$. The organic layer was dried over $\mathrm{Na}_{2} \mathrm{SO}_{4}$, concentrated, and dried in vacuo to afford crude 2,3,4,6-tetra-0-butyryl-a-D-glucopyranosyl bromide 6 (3.7 $\mathrm{g}, 70 \%)$. The crude butyryl-glucopyranosyl bromide $6(3.7 \mathrm{~g}, 7.1 \mathrm{mmol})$ was dissolved in DMF $(5 \mathrm{~mL})$ and sodium azide $(690 \mathrm{mg}, 10.6 \mathrm{mmol}$ ) was added to the resulting solution. The resulting mixture was stirred at $50{ }^{\circ} \mathrm{C}$ for 12 hours until no starting material was detected using TLC analysis. The reaction mixture was diluted with $\mathrm{CH}_{2} \mathrm{Cl}_{2}(2 \mathrm{~mL})$, and then washed with aqueous saturated $\mathrm{NaHCO}_{3}$. The organic layer was dried using $\mathrm{Na}_{2} \mathrm{SO}_{4}$ and evaporated, and the resulting residue was purified by column chromatography in silica gel (petroleum ether $60^{\circ} \mathrm{C}-90^{\circ} \mathrm{C}$ : ethyl acetate $=9: 1$ ) to afford 2,3,4,6-tetra-0butyryl-1-azido-a-D-glucopyranose 7 (2.1 g, 60\%).

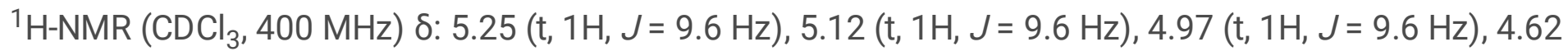
$\left(\mathrm{d}, 1 \mathrm{H}, J=8.6 \mathrm{~Hz}, \mathrm{C}^{1}-\mathrm{H}\right), 4.21-4.19(\mathrm{~m}, 2 \mathrm{H}), 3.76-3.72(\mathrm{~m}, 1 \mathrm{H}), 2.35-2.19\left(\mathrm{~m}, 8 \mathrm{H}, 4 \times \mathrm{COCH}_{2}\right), 1.68-1.53$ $\left(\mathrm{m}, 8 \mathrm{H}, 4 \times \underline{\mathrm{CH}}_{2} \mathrm{CH}_{3}\right), 0.96-0.87\left(\mathrm{~m}, 12 \mathrm{H}, 4 \times \mathrm{CH}_{2} \underline{\mathrm{CH}}_{3}\right) ;{ }^{13} \mathrm{C}-\mathrm{NMR}\left(\mathrm{CDCl}_{3}, 100 \mathrm{MHz}\right) \delta: 173.3(\mathrm{C}=0), 172.6$ $(\mathrm{C}=0), 171.9(\mathrm{C}=0), 171.9(\mathrm{C}=0), 88.0,74.2,72.2,70.4,67.7,61.5,35.8\left(\mathrm{COCH}_{2}\right), 35.8\left(\mathrm{COCH}_{2}\right), 35.7$ $\left(\mathrm{COCH}_{2}\right), 35.7\left(\mathrm{COCH}_{2}\right), 18.2\left(\underline{\mathrm{CH}}_{2} \mathrm{CH}_{3}\right), 18.2\left(\underline{\mathrm{CH}}_{2} \mathrm{CH}_{3}\right), 18.2\left(\underline{\mathrm{CH}}_{2} \mathrm{CH}_{3}\right), 18.1\left(\underline{\mathrm{CH}}_{2} \mathrm{CH}_{3}\right), 13.6\left(\mathrm{CH}_{2} \underline{\mathrm{CH}_{3}}\right)$, $13.5\left(\mathrm{CH}_{2} \underline{\mathrm{CH}}_{3}\right), 13.5\left(\mathrm{CH}_{2} \underline{\mathrm{CH}}_{3}\right), 13.5\left(\mathrm{CH}_{2} \underline{\mathrm{CH}}_{3}\right) ; \mathrm{ESIMS} \mathrm{m} / z 508[\mathrm{M}+\mathrm{Na}]^{+}$.

\section{Synthesis of 0-alkylated (-)-epigallocatechin-3-gallate conjugates 8 and 9}


A mixture of EGCG $(2.3 \mathrm{~g}, 5 \mathrm{mmol})$, DMF $(15 \mathrm{~mL})$ and $\mathrm{K}_{2} \mathrm{CO}_{3}(1.0 \mathrm{~g}, 7.5 \mathrm{mmol})$ was made at room temperature under nitrogen and stirred for $0.5 \mathrm{~h}$. Propargyl bromide $(0.3 \mathrm{~mL}, 7.5 \mathrm{mmol})$ was quickly added to the resulting mixture, which was then stirred at room temperature for $12 \mathrm{~h}$ until no starting material was detected according to TLC analysis. The solvent of the resulting mixture was evaporated under vacuum and the residue was purified using column chromatography with silica gel $\left(\mathrm{CHCl}_{3} / \mathrm{CH}_{3} \mathrm{OH}\right.$, $9: 1 \rightarrow 4: 1)$ to afford the EGCG conjugates $8(1.1 \mathrm{~g}, 45 \%)$ and $\mathbf{9}(0.8 \mathrm{~g}, 30 \%)$.

\section{5,7-Dihydroxy-2-(3',4',5'-trihydroxyphenyl)chroman-3-yl 3",5"-dihydroxy-4"-}

(prop-2-yn-1-yloxy)benzoate (8)

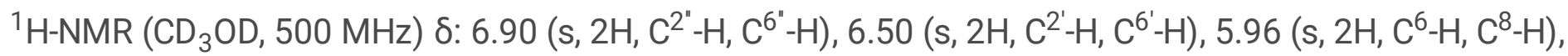
$5.53\left(\mathrm{brs}, 1 \mathrm{H}, \mathrm{C}^{3}-\mathrm{H}\right), 4.97\left(\mathrm{~s}, 1 \mathrm{H}, \mathrm{C}^{2}-\mathrm{H}\right), 4.78\left(\mathrm{~d}, 2 \mathrm{H} \mathrm{J}=2.4 \mathrm{~Hz}, \mathrm{C}^{12}-\mathrm{CH}_{2}\right), 3.29\left(\mathrm{t}, 1 \mathrm{H}, J=1.6 \mathrm{~Hz}, \mathrm{C}^{14}-\mathrm{H}\right), 2.96$ $\left(\mathrm{dd}, 1 \mathrm{H}, J=4.6 \mathrm{~Hz}, 12.0 \mathrm{~Hz}, \mathrm{C}^{4}-\mathrm{CH}_{\mathrm{a}}\right), 2.85\left(\mathrm{dd}, 1 \mathrm{H}, J=4.6 \mathrm{~Hz}, 12.0 \mathrm{~Hz}, \mathrm{C}^{4}-\mathrm{CH}_{\mathrm{b}}\right) ;{ }^{13} \mathrm{CNMR}\left(\mathrm{CD}_{3} \mathrm{OD}, 125\right.$ $\mathrm{MHz}$ ) ठ: 167.0 (C=0), 157.9 (C-7), 157.8 (C-5), 157.2 (C-9), 151.9 (C-3", C-5"), 146.7 (C-3', C-5'), 138.4 (C-4"), 133.8 (C-4), 130.7 (C-1'), 127.1 (C-1"), 110.1 (C-2", C-6'), 106.8 (C-2', C-6'), 99.3 (C-10), 96.5 (C-6), 95.9 (C-8), $80.4(\mathrm{C}-2), 79.5(\underline{\mathrm{C}} \equiv \mathrm{CH}), 78.5(\mathrm{C} \equiv \underline{\mathrm{CH}}), 70.3(\mathrm{C}-3), 60.0$ (C-14), $26.8(\mathrm{C}-4)$; ESIMS m/z $495\left[\mathrm{M}-\mathrm{H}^{-}\right.$.

\section{5,7-Dihydroxy-2-(3',5'-dihydroxy-4'-(prop-2-yn-1-yloxy)phenyl)-3",5"-dihydroxy-4"-(prop-2-yn-1- yloxy)benzoate (9)}

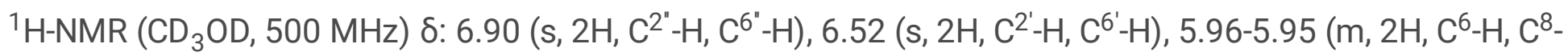
$\mathrm{H})$, 5.56-5.55 (m, $\left.1 \mathrm{H}, \mathrm{C}^{3}-\mathrm{H}\right), 5.00\left(\mathrm{~s}, 1 \mathrm{H}, \mathrm{C}^{2}-\mathrm{H}\right), 4.77\left(\mathrm{~d}, 2 \mathrm{H}, J=2.4 \mathrm{~Hz}, \mathrm{OCH}_{2}\right), 4.67\left(\mathrm{~d}, 2 \mathrm{H}, J=2.4 \mathrm{~Hz}, \mathrm{OCH}_{2}\right)$, $3.33(\mathrm{~s}, 2 \mathrm{H}, 2 \times \mathrm{C} \equiv \mathrm{CH}), 2.98\left(\mathrm{dd}, 1 \mathrm{H}, J=4.6 \mathrm{~Hz}, 12.0 \mathrm{~Hz}, \mathrm{C}^{4}-\mathrm{CH}_{\mathrm{a}}\right), 2.88\left(\mathrm{~m}, 1 \mathrm{H}, \mathrm{C}^{4}-\mathrm{CH}_{\mathrm{b}}\right) ;{ }^{13} \mathrm{CNMR}\left(\mathrm{CD}_{3} \mathrm{OD}\right.$, $125 \mathrm{MHz}$ ) ठ: 167.0 (C=0), 157.9 (C-7), 157.8 (C-5), 157.0 (C-9), 151.9 (C-3", C-5"), 151.7 (C-3', C-5), 138.4 (C-4"), 136.3 (C-4), 133.7 (C-1'), 127.0 (C-1"), 110.1 (C-2", C-6"), 106.8 (C-2', C-6), 99.3 (C-10), 96.6 (C-6), 95.9 (C-8), $80.4(\underline{\mathrm{C}} \equiv \mathrm{CH}), 80.0(\underline{\mathrm{C}} \equiv \mathrm{CH}), 78.2(\mathrm{C}-2), 76.7(\mathrm{C} \equiv \underline{\mathrm{CH}}), 76.4(\mathrm{C} \equiv \underline{\mathrm{CH}}), 70.2(\mathrm{C}-3), 60.2\left(\mathrm{OCH}_{2}\right), 60.0$ $\left(\mathrm{OCH}_{2}\right), 26.8(\mathrm{C}-4) ; \mathrm{ESIMS} \mathrm{m} / \mathrm{z} 533[\mathrm{M}-\mathrm{H}]^{-}$.

General procedure for the synthesis of 2,3,4,6-tetra-0-butyryl-a-D-glucopyranosyloxy-1,2,3-triazol-1-yl-(-)epigallocatechin-3-gallates (10 and 11)

For each of the two target compounds, a solution of compound $7(0.1 \mathrm{mmol})$ and 0 -alkylated (-)epigallocatechin-3-gallate conjugates 8 or $9(0.1 \mathrm{mmol})$ in THF $(1.0 \mathrm{~mL})$ and ${ }^{t}{ }^{t} \mathrm{BuOH}-\mathrm{H}_{2} \mathrm{O}(1.0 \mathrm{~mL}, 1: 1)$ was prepared, and copper(II) acetate $(0.01 \mathrm{mmol})$ and sodium ascorbate $(0.01 \mathrm{mmol})$ were added to this solution. This reaction mixture was stirred at room temperature for $2 \mathrm{~h}$ until no starting material was detected according to TLC analysis. The resulting mixture was evaporated under vacuum and the residue was purified using column chromatography with silica gel $\left(\mathrm{CHCl}_{3} / \mathrm{CH}_{3} \mathrm{OH}, 9: 1\right)$ to afford the target cycloaddition product. 
4"-0-[(2"',3"',4"',6"'-tetra-0-butyryl-a-D-glucopyranosyloxy)-1,2,3-triazol-1-yl)tetrahydro-2H-pyran]-(-)epigallocatechin-3-gallate (10)

White amorphous powder; Yield: 80\%; MP. 98-100 ${ }^{\circ} \mathrm{C}$; [a]24.1 D: +14.2 (c 0.22, $\left.\mathrm{CH}_{3} \mathrm{OH}\right) ;{ }^{1} \mathrm{H}-\mathrm{NMR}\left(\mathrm{CD}_{3} \mathrm{OD}\right.$,

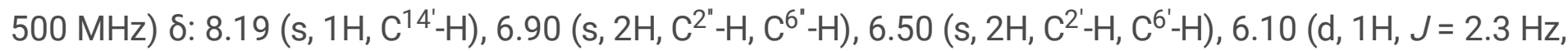
$\left.\mathrm{C}^{1 " '}-\mathrm{H}\right), 5.94\left(\mathrm{~s}, 2 \mathrm{H}, \mathrm{C}^{6}-\mathrm{H}, \mathrm{C}^{8}-\mathrm{H}\right), 5.61(\mathrm{t}, 1 \mathrm{H}, J=9.4 \mathrm{~Hz}), 5.53-5.50(\mathrm{~m}, 2 \mathrm{H}), 5.27-5.24(\mathrm{~m}, 1 \mathrm{H}), 5.23(\mathrm{~s}, 2 \mathrm{H}$, $\left.\mathrm{C}^{12}-\mathrm{CH}_{2}\right), 4.96\left(\mathrm{~s}, 1 \mathrm{H}, \mathrm{C}^{2}-\mathrm{H}\right), 4.36-4.18(\mathrm{~m}, 3 \mathrm{H}), 2.98\left(\mathrm{dd}, 1 \mathrm{H}, J=4.6 \mathrm{~Hz}, 12.1 \mathrm{~Hz}, \mathrm{C}^{4}-\mathrm{H}_{\mathrm{a}}\right), 2.88-2.86(\mathrm{~m}$, $\left.1 \mathrm{H}, \mathrm{C}^{4}-\mathrm{H}_{\mathrm{b}}\right), 2.23-1.94\left(\mathrm{~m}, 8 \mathrm{H}, 4 \times \mathrm{COCH}_{2}\right), 1.60-1.26\left(\mathrm{~m}, 8 \mathrm{H}, 4 \times \underline{\mathrm{CH}}_{2} \mathrm{CH}_{3}\right), 0.93-0.62(\mathrm{~m}, 12 \mathrm{H}, 4 \times$

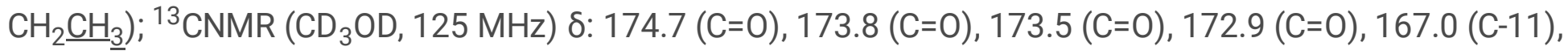
157.9 (C-7), 157.8 (C-5), 157.2 (C-9), 151.6 (C-3", C-5"), 146.6 (C-3', C-5'), 145.8 (C-14'), 138.8 (C-4"), 133.7 (C-4), 130.7 (C-1'), 126.9 (C-13'), 124.8 (C-1'), 110.3 (C-2", C-6'), 106.7 (C-2', C-6'), 99.2 (C-10), 96.5 (C-6), 95.8 (C-8), 86.4 (C-1"'), 78.5 (C-2), 75.9, 74.0, 71.4, 70.3 (C-12'), 68.9, $65.3(\mathrm{C}-3), 62.6,36.7\left(\mathrm{CO}^{\mathrm{CH}} \underline{2}_{2}\right), 36.6$ $\left(\mathrm{COCH}_{2}\right), 36.6\left(\mathrm{COCH}_{2}\right), 36.2\left(\mathrm{COCH}_{2}\right), 26.8(\mathrm{C}-4), 19.2\left(\underline{\mathrm{CH}}_{2} \mathrm{CH}_{3}\right), 19.2\left(\underline{\mathrm{CH}}_{2} \mathrm{CH}_{3}\right), 19.2\left(\underline{\mathrm{CH}}_{2} \mathrm{CH}_{3}\right), 19.0$ $\left(\mathrm{CH}_{2} \mathrm{CH}_{3}\right), 13.9\left(\mathrm{CH}_{2} \underline{\mathrm{CH}}_{3}\right), 13.9\left(\mathrm{CH}_{2} \underline{\mathrm{CH}}_{3}\right), 13.9\left(\mathrm{CH}_{2} \underline{\mathrm{CH}}_{2}\right), 13.6\left(\mathrm{CH}_{2} \underline{\mathrm{CH}}_{3}\right) ; \mathrm{ESIMS} \mathrm{m} / \mathrm{z} 980[\mathrm{M}-\mathrm{H}]^{-}$, HRESIMS was calculated for $\mathrm{C}_{47} \mathrm{H}_{55} \mathrm{~N}_{3} \mathrm{O}_{20}[\mathrm{M}-\mathrm{H}]^{-} 980.3306$ and was found to be 980.3306 .

\section{$\{4$-0-[(2"',3"',4"',6"'-tetra-0-butyryl-a-D-glucopyranosyloxy)-1,2,3-triazol-1-yl)tetrahydro-2H-pyran]-4"-0- [(2"',3"','4"',6"'-tetra-0-butyryl-a-D-glucopyranosyloxy)-1,2,3-triazol-1-yl)tetrahydro-2H-pyran]\}-(-)- epigallocatechin-3-gallate (11)}

White amorphous powder; Yield: 77\%; MP: $92-93^{\circ} \mathrm{C}$; [a]24.1 D: +19.3 (c 0.14, $\left.\mathrm{CH}_{3} \mathrm{OH}\right) ;{ }^{1} \mathrm{H}-\mathrm{NMR}\left(\mathrm{CD}_{3} \mathrm{OD}\right.$,

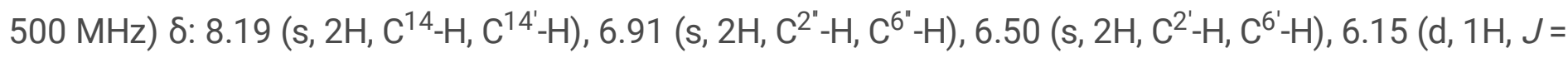
$\left.2.3 \mathrm{~Hz}, \mathrm{C}^{1 "}-\mathrm{H}\right), 6.10\left(\mathrm{~d}, 1 \mathrm{H}, J=9.3 \mathrm{~Hz}, \mathrm{C}^{1 "}-\mathrm{H}\right), 5.94\left(\mathrm{~s}, 2 \mathrm{H}, \mathrm{C}^{6}-\mathrm{H}, \mathrm{C}^{8}-\mathrm{H}\right), 5.61(\mathrm{t}, 2 \mathrm{H}, J=9.4 \mathrm{~Hz}), 5.56-5.53$ $(\mathrm{m}, 3 \mathrm{H}), 5.29-5.26(\mathrm{~m}, 2 \mathrm{H}), 5.20-5.15(\mathrm{~m}, 4 \mathrm{H}), 4.96\left(\mathrm{~s}, 1 \mathrm{H}, \mathrm{C}^{2}-\mathrm{H}\right), 4.38-4.36(\mathrm{~m}, 2 \mathrm{H}), 4.22-4.18(\mathrm{~m}, 2 \mathrm{H})$, 3.10-3.06 (m, $\left.2 \mathrm{H}, \mathrm{C}^{4}-\mathrm{CH}_{2}\right), 2.24-2.00\left(\mathrm{~m}, 16 \mathrm{H}, 8 \times \mathrm{CO}_{\underline{\mathrm{C}}}\right), 1.70-1.49\left(\mathrm{~m}, 16 \mathrm{H}, 8 \times \underline{\mathrm{CH}}_{2} \mathrm{CH}_{3}\right), 1.01-0.87$ $\left(\mathrm{m}, 24 \mathrm{H}, 8 \times \mathrm{CH}_{2} \underline{\mathrm{CH}}_{3}\right) ;{ }^{13} \mathrm{C}-\mathrm{NMR}\left(\mathrm{CD}_{3} \mathrm{OD}, 125 \mathrm{MHz}\right) \delta: 174.3(\mathrm{C}=0), 174.2(\mathrm{C}=0), 174.1(\mathrm{C}=0), 174.1(\mathrm{C}=0)$, $174.0(\mathrm{C}=0), 174.0(\mathrm{C}=0), 173.9$ (C=0), $173.7(\mathrm{C}=0), 167.0$ (C-11), 157.9 (C-7), 157.8 (C-5), 157.2 (C-9), 151.6 (C-3", C-5"), 146.6 (C-3', C-5'), 145.8 (C-14), 145.8 (C-14'), 138.8 (C-4"), 133.7 (C-4'), 130.7 (C-1'), 126.9 (C-13), 126.9 (C-13'), 124.8 (C-1"), 110.3 (C-2", C-6'), 106.7 (C-2', C-6'), 99.2 (C-10), 96.5 (C-6), 95.8 (C-8), 86.6 (C-1"'), 86.4 (C-1"), 78.5 (C-2), 77.0, 74.1, 73.6, 73.1, 72.8, 72.5, 71.8, 71.5, 69.8 (C-12), 69.5 (C-12), $66.9(\mathrm{C}-3), 62.6,62.6,36.9\left(\mathrm{COCH}_{2}\right), 36.9\left(\mathrm{COCH}_{2}\right), 36.8\left(\mathrm{COCH}_{2}\right), 36.8\left(\mathrm{COCH}_{2}\right), 36.7\left(\mathrm{COCH}_{2}\right), 36.7$ $\left(\mathrm{COCH}_{2}\right), 36.6\left(\mathrm{CO}_{\underline{\mathrm{CH}}}\right), 36.6\left(\mathrm{CO}_{\underline{\mathrm{CH}}}\right), 26.8(\mathrm{C}-4), 19.5\left(\underline{\mathrm{CH}}_{\underline{2}} \mathrm{CH}_{3}\right), 19.5\left(\underline{\mathrm{CH}}_{2} \mathrm{CH}_{3}\right), 19.4\left(\underline{\mathrm{CH}}_{2} \mathrm{CH}_{3}\right), 19.4$ $\left(\underline{\mathrm{CH}}_{2} \mathrm{CH}_{3}\right), 19.3\left(\underline{\mathrm{CH}}_{2} \mathrm{CH}_{3}\right), 19.2\left(\underline{\mathrm{CH}}_{2} \mathrm{CH}_{3}\right), 19.2\left(\underline{\mathrm{CH}}_{2} \mathrm{CH}_{3}\right), 19.1\left(\underline{\mathrm{CH}}_{2} \mathrm{CH}_{3}\right), 14.2\left(\mathrm{CH}_{2} \underline{\mathrm{CH}_{3}}\right), 14.1\left(\mathrm{CH}_{2} \underline{\mathrm{CH}}_{3}\right)$, $14.0\left(\mathrm{CH}_{2} \underline{\mathrm{CH}}_{3}\right), 14.0\left(\mathrm{CH}_{2} \underline{\mathrm{CH}}_{3}\right), 13.9\left(\mathrm{CH}_{2} \underline{\mathrm{CH}}_{3}\right), 13.9\left(\mathrm{CH}_{2} \underline{\mathrm{CH}}_{3}\right), 13.9\left(\mathrm{CH}_{2} \underline{\mathrm{CH}}_{\underline{3}}\right), 13.7\left(\mathrm{CH}_{2} \underline{\mathrm{CH}}_{\underline{3}}\right) ; \mathrm{ESIMS} \mathrm{m} / z$ $1503[\mathrm{M}-\mathrm{H}]^{-}$, HRESIMS was calculated for $\mathrm{C}_{72} \mathrm{H}_{91} \mathrm{~N}_{6} \mathrm{O}_{29}[\mathrm{M}-\mathrm{H}]^{-} 1503.5946$ and was found to be 1503.5939 . 
General procedure for the syntheses of a-D-glucopyranosyloxy-1,2,3-triazol-1-yl-(-)-epigallocatechin-3gallates (12 and 13)

For each of the target compounds, a solution of 2,3,4,6-tetra-O-butyryl-a-D-glucopyranosyloxy-1,2,3-triazol1-yl-(-)-epigallocatechin-3-gallate (100r 11) $(0.05 \mathrm{mmol})$ in $\mathrm{CH}_{3} \mathrm{OH}(1 \mathrm{~mL})$ was prepared, and to this solution was added a $\mathrm{KOH}$ solution $\left(0.15 \mathrm{mmol}\right.$, dissolved in $\left.\mathrm{CH}_{3} \mathrm{OH}\right)$. This mixture was stirred at $0{ }^{\circ} \mathrm{C}$ for $72 \mathrm{~h}$, and then neutralized with Dowex 50WX4-400 ion-exchange resin to $\mathrm{pH}=7$. The solvent of the resulting mixture was evaporated in vacuum and resulting the residue was purified using column chromatography with silica gel $\left(\mathrm{CHCl}_{3} / \mathrm{CH}_{3} \mathrm{OH}, 4: 1\right)$ to afford the product.

\section{4"-0-[(1"'--a-D-glucopyranosyloxy)-1,2,3-triazol-1-yl)tetrahydro-2H-pyran]-(-)-epigallocatechin-3-gallate (12)}

White amorphous powder; Yield: 55\%; MP: $125-126^{\circ} \mathrm{C}$; [a]23.8 D: +14.4 (c $\left.0.23, \mathrm{CH}_{3} \mathrm{OH}\right) ;{ }^{1} \mathrm{HNMR}\left(\mathrm{CD}_{3} \mathrm{OD}\right.$,

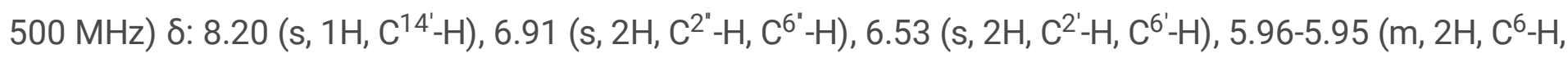

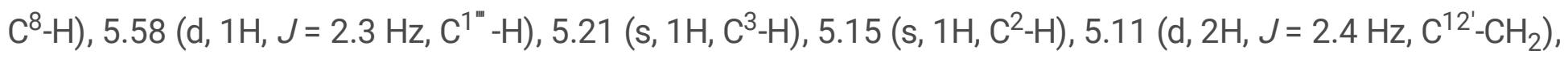
3.89-3.86 (m, 2H), 3.72-3.69 (m, 1H), 3.57-3.53 (m, 2H), 3.51-3.48 (m, 1H), 2.99-2.87 (m, 1H, $\left.\mathrm{C}^{4}-\mathrm{CH}_{\mathrm{a}}\right)$,

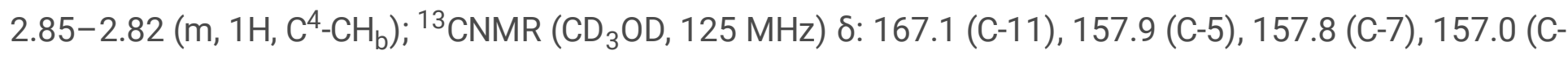
9), 151.9 (C-3", C-5'), 151.7 (C-3', C-5'), 146.7 (C-14'), 139.4 (C-4'), 136.3 (C-4'), 127.0 (C-1'), 124.9 (C-1'), 124.9 (C-13'), 110.4 (C-2', C-6"), 106.9 (C-2', C-6'), 99.3 (C-10), 96.6 (C-6), 95.9 (C-8), 89.6 (C-1"), 81.1 (C-2), 78.4, 74.1, 70.8, 70.2 (C-12), 66.0, 62.4 (C-3), 60.0, 26.7 (C-4); ESIMS: $m / z=702$ [M + H] , HRESIMS was calculated for $\mathrm{C}_{31} \mathrm{H}_{31} \mathrm{~N}_{3} \mathrm{O}_{16}\left[\mathrm{M}-\mathrm{H}^{-} 700.1632\right.$ and was found to be 700.1636 .

\section{\{4'-0-[(1"'-a-D-glucopyranosyloxy)-1,2,3-triazol-1-yl)tetrahydro-2H-pyran]-4"-0-[(1'"-a-D-} glucopyranosyloxy)-1,2,3-triazol-1-yl)tetrahydro-2H-pyran]-(-)-epigallocatechin-3-gallate (13)

White amorphous powder; Yield: 52\%; MP: $120-121^{\circ} \mathrm{C}$; [a]25.0 D: +22.4 (c 0.24, $\left.\mathrm{CH}_{3} \mathrm{OH}\right) ;{ }^{1} \mathrm{HNMR}\left(\mathrm{CD}_{3} \mathrm{OD}\right.$,

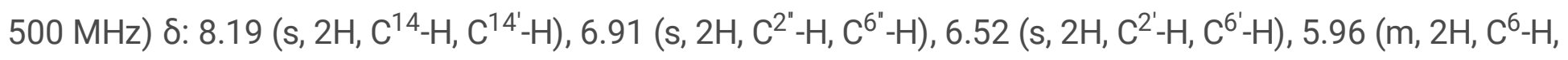
$\left.\mathrm{C}^{8}-\mathrm{H}\right), 5.59\left(\mathrm{~d}, 1 \mathrm{H}, J=1.6 \mathrm{~Hz}, \mathrm{C}^{1{ }^{\prime \prime}}-\mathrm{H}\right), 5.57\left(\mathrm{~d}, 1 \mathrm{H}, J=1.6 \mathrm{~Hz}, \mathrm{C}^{1 "}-\mathrm{H}\right), 5.58\left(\mathrm{~s}, 1 \mathrm{H}, \mathrm{C}^{3}-\mathrm{H}\right), 5.21\left(\mathrm{~s}, 2 \mathrm{H}, \mathrm{C}^{12}-\right.$ $\left.\mathrm{CH}_{2}\right), 5.11\left(\mathrm{~s}, 2 \mathrm{H}, \mathrm{C}^{12}-\mathrm{CH}_{2}\right), 5.00\left(\mathrm{~s}, 1 \mathrm{H}, \mathrm{C}^{2}-\mathrm{H}\right), 3.90-3.85(\mathrm{~m}, 4 \mathrm{H}), 3.71-3.68(\mathrm{~m}, 2 \mathrm{H}), 3.57-3.54(\mathrm{~m}, 4 \mathrm{H})$, 3.53-3.52 (m, 2H), $2.98\left(\mathrm{dd}, 1 \mathrm{H}, J=4.6 \mathrm{~Hz}, 12.1 \mathrm{~Hz}, \mathrm{C}^{4}-\mathrm{H}_{\mathrm{a}}\right), 2.88-2.86\left(\mathrm{~m}, 1 \mathrm{H}, \mathrm{C}^{4}-\mathrm{H}_{\mathrm{b}}\right) ;{ }^{13} \mathrm{CNMR}\left(\mathrm{CD}_{3} \mathrm{OD}\right.$, $125 \mathrm{MHz}$ ) ठ: 166.9 (C-11), 157.9 (C-7), 157.8 (C-5), 157.0 (C-9), 151.7 (C-3", C-5'), 151.5 (C-3', C-5'), 145.5 (C-13), 145.4 (C-14'), 139.4 (C-4"), 136.3 (C-4'), 134.5 (C-1'), 126.9 (C-1"), 124.8 (C-14), 124.7 (C-13'), 110.4 (C-2", C-6"), 107.1 (C-2', C-6'), 99.2 (C-10), 96.6 (C-6), 95.8 (C-8), 89.6 (C-1"'), 89.5 (C-1"), 81.1 (C-2), 78.4 , 78.3, 78.3, 78.2, 74.1, 74.0, 70.8 (C-12), 70.8 (C-12), 70.2 (C-3), 66.0, 65.8, 62.4, 62.3, 26.7 (C-4); ESIMS: $\mathrm{m} / \mathrm{z}=945[\mathrm{M}+\mathrm{H}]^{+}$, HRESIMS was calculated for $\mathrm{C}_{40} \mathrm{H}_{44} \mathrm{~N}_{6} \mathrm{O}_{21}[\mathrm{M}-\mathrm{H}]^{-} 943.2487$ and was found to be 943.2460 .

\section{Cytotoxicity Assay}


MTT assays were conducted to evaluate the cell viabilities of the triazole-linked glucose-(-)epigallocatechin-3-gallate derivatives. Cells of five human cancer lines, namely HL-60, SMMC-7721, A549, MCF-7 and SW480, were seeded in 96-well plates and then exposed to the test compound at various concentrations in triplicate for $48 \mathrm{~h}$. After the incubation, MTT $(100 \mu \mathrm{g})$ was added to each well, and the incubation was continued for $4 \mathrm{~h}$ at $37^{\circ} \mathrm{C}$. After removal of the culture medium, the produced MTT formazan crystals were dissolved in DMSO $(150 \mu \mathrm{L})$ and the OD of the resulting solution was measured at a wavelength of $492 \mathrm{~nm}$ using a microplate reader. The percent inhibition was calculated using the formula inhibition ratio $(\mathrm{IR}, \%)=(1-\mathrm{OD}$ (sample) $/ \mathrm{OD}$ (control) $) \times 100 \%$. The experiments were carried out in triplicate, and the $\mathrm{IC}_{50}$ (the concentration of drug that inhibits cell growth by $50 \%$ ) values were determined.

\section{Cell Apoptosis Assay}

An annexin V/propidium iodide (PI) detection kit (BD Biosciences, PA, USA) was employed to quantify apoptosis using flow cytometry. For each experiment, HL-60 cells were seeded into each well of a 6-well plate at $5 \times 10^{5}$ cells/well and then treated with one of the EGCG derivatives. After the treatment, the collected cells were incubated in $100 \mu \mathrm{L}$ of binding buffer, and then into the resulting suspension were added $5 \mu \mathrm{L}$ of FITC annexin V and $10 \mu \mathrm{L}$ of Pl. Each mixture was gently vortexed and then incubated for $15 \mathrm{~min}$ at room temperature in the dark before taking the flow cytometry measurements (BD FACSCalibur) within $1 \mathrm{~h}$.

\section{Calculated Partition Coefficient}

All structures of EGCG derivatives were built and energy minimized by applying the Tripos force field. The Gasteiger-Huchel method was used to calculate charges. Energy minimization was performed by applying the Powell method with 2000 iterations. The calculated partition coefficient (ClogP) was obtained from MarvinSketch version 5.3.8. (www.chemaxon.org) [29].

\section{Water Solubility Analysis}

All compounds were subjected to water solubility analyses. Each compound was mixed in $200 \mu \mathrm{L}$ of water in an Eppendorf tube at room temperature. An ultrasonic cleaner was used to maximize the amount of the compound that became dissolved. After $1 \mathrm{~h}$ of sonication, each sample was diluted and then filtered through a $0.45 \mu \mathrm{m}$ MFS membrane for HPLC analysis to determine the concentrations of the tested compound [21]. Analytical HPLC was performed using an Agilent 1260 liquid chromatograph equipped with a ZORBAX SB-C18 $(4.6 \times 250 \mathrm{~mm})$ column. Here, the detection wavelength was $280 \mathrm{~nm}$, the injection volume was $10 \mu \mathrm{L}$. The flow rate was $1 \mathrm{~mL} / \mathrm{min}$.

\section{Declarations}

Acknowledgements The authors are grateful for financial supports from National Natural Science Foundation of China (Grant No. 31960075, 21602196, 31760226); the Yunnan Provincial Science and 
Technology Department (Grant No. 2017ZF003, 2017FD084, 2017FG001-046); and the Yunnan Provincial Key Programs of Yunnan Eco-friendly Food International Cooperation Research Center Project (Grant No. 2019ZG00904, 2019ZG00909).

\section{Compliance with ethical standards}

Conflict of Interests All authors declare that: (i) except for National Natural Science Foundation of China, the Yunnan Provincial Science and Technology Department and the Yunnan Provincial Key Programs of Yunnan Eco-friendly Food International Cooperation Research Center Project, no support, financial or otherwise, has been received from any other organization that may have an interest in the submitted work; and (ii) there are no other relationships or activities that could appear to have influenced the submitted work.

Publisher's note Springer Nature remains neutral with regard to jurisdictional claims in published maps and institutional affiliations.

\section{References}

1. Ahmed S, Stepp JR Green Tea: The Plants, Processing, Manufacturing and Production. Tea in Health and Disease Prevention. 2012.

2. Chen J, Wang PS, Xia YM, Xu M, Pei SJ. Genetic diversity and differentiation of Camellia sinensis L. (cultivated tea) and its wild relatives in Yunnan province of China, revealed by morphology, biochemistry and allozyme studies. Genet Resour Crop Ev. 2005;52:41-52. doi:10.1007/s10722-0050285-1

3. Yang CS, Wang ZY. Tea and cancer. J Natl Cancer Inst. 1993;85:1038-49. doi:10.1093/jnci/85.13.1038

4. Yang CS, Lambert JD, Sang S. Antioxidative and anti-carcinogenic activities of tea polyphenols. Arch Toxicol. 2009;83:11-21. doi:10.1007/s00204-008-0372-0

5. Yuan JM, Sun CL, Butler LM. Tea and cancer prevention: Epidemiological studies. Pharmacol Res. 2011;64:123-35. doi:10.1016/j.phrs.2011.03.002

6. Yang Y, Qiao LL, Zhang X, Wu Z, Weng P. Effect of methylated tea catechins from Chinese oolong tea on the proliferation and differentiation of 3T3-L1 preadipocyte. Fitoterapia 2015;104:45-9. doi:10.1016/j.fitote.2015.05.007

7. Cabrera C, Giménez R, López MC. Determination of tea components with antioxidant activity. J Agric Food Chem. 2003;51:4427-35. doi:10.1021/jf0300801

8. Jung YD, Kim MS, Shin BA, Chay KO, Ahn BW, Liu W, et al. EGCG, a major component of green tea, inhibits tumour growth by inhibiting VEGF induction in human colon carcinoma cells. Br J Cancer 2001;84:844-50. doi:10.1054/bjoc.2000.1691

9. Wang J, Tang H, Hou B, Zhang P, Wang Q, Zhang BL, et al. Synthesis, antioxidant activity, and density functional theory study of catechin derivatives. RSC Advances 2017;7:54136-141. 
doi:10.1039/C7RA11496F

10. Steinmann J, Buer J, Pietschmann T, Steinmann E. Anti-infective properties of epigallocatechin-3gallate (EGCG), a component of green tea. Br J Pharmacol. 2013;168:1059-73.

doi:10.1111/bph.12009

11. Huo C, Wan SB, Lam WH, Li L, Wang Z, Landis-Piwowar KR, et al. The challenge of developing green tea polyphenols as therapeutic agents. Inflammopharmacology 2008;16:248-52. doi:10.1007/s10787-008-8031-x

12. Hugel HM, Jackson N. Redox Chemistry of Green Tea Polyphenols: Therapeutic Benefits in Neurodegenerative Diseases. Mini-Rev Med Chem. 2012;12:380-387.

doi:10.2174/138955712800493906

13. Kitao S, Matsudo T, Sasaki M, Horiuchi T, Sekine H. Enzymatic Syntheses of Two Stable (-)Epigallocatechin Gallate-glucosides by Sucrose Phosphorylase. Biosci Biotechnol Biochem. 1995;59:2167-9. doi:10.1271/bbb.59.2167

14. Hong J, Lu H, Meng X, Ryu JH, Yang CS. Stability, cellular uptake, biotransformation, and efflux of tea polyphenol (-)-epigallocatechin-3-gallate in HT-29 human colon adenocarcinoma cells. Cancer Res. 2002;62:7241-6. doi:10.1016/S0304-3835(02)00435-4

15. Wong ILK, Wang BC, Yuan J, Duan LX, Liu Z, Liu T, et al. Potent and Nontoxic Chemosensitizer of Pglycoprotein-mediated Multidrug Resistance in Cancer: Synthesis and Evaluation of Methylated Epigallocatechin, Gallocatechin, and Dihydromyricetin Derivatives. J Med Chem. 2015;58:4529-49. doi:10.1021/acs.jmedchem.5b00085

16. Qsanai K, Landis-Piwowar KR, Dou QP, Chan TH. A para-amino substituent on the D-ring of green tea polyphenol epigallocatechin-3-gallate as a novel proteasome inhibitor and cancer cell apoptosis inducer. Bioorg Med Chem. 2007;15:5076-82. doi:10.1016/j.bmc.2007.05.041

17. Mori S, Miyake S, Kobe T, Nakaya T, Fuller SD, Kato N, et al. Enhanced anti-influenza A virus activity of (-)-epigallocatechin-3-O-gallate fatty acid monoester derivatives: Effect of alkyl chain length. Bioorg Med Chem Lett. 2008;18:4249-52. doi:10.1016/j.bmcl.2008.02.020

18. Moon YH, Lee JH, Ahn JS, Nam SH, Oh DK, Park DH, et al. Synthesis, structure analyses, and characterization of novel epigallocatechin gallate (EGCG) glycosides using the glucansucrase from Leuconostoc mesenteroides B-1299CB. J Agric Food Chem. 2006;54:1230-7. doi:10.1021/jf052359i

19. Kitao S, Ariga T, Matsudo T, Sekine H. The syntheses of catechin-glucosides by transglycosylation with Leuconostoc mesenteroides sucrose phosphorylase. Biosci Biotechnol Biochem. 1993;57:2010-5. doi:10.1271/bbb.57.2010

20. Sato T, Nakagawa H, Kurosu J, Yoshida K, Tsugane T, Shimura S, et al. a-Anomerselective glucosylation of (+)-catechin by the crude enzyme, showing glucosyl transfer activity, of Xanthomonas campestris WU-9701. J Biosci Bioeng. 2000;90:625-30. doi:10.1016/S13891723(00)90007-0

21. Zhang X, Wang J, Hu JM, Huang YW, Wu XY, Zi CT, et al. Synthesis and biological testing of novel glucosylated epigallocatechin gallate (EGCG) derivatives. Molecules 2016;21:620. 
doi:10.3390/molecules21050620

22. Calvaresi EC, Hergenrother PJ. Glucose conjugation for the specific targeting and treatment of cancer. Chem Sci. 2013;4:2319-33. doi:10.1039/c3sc22205e

23. Arafa HMM. Possible contribution of beta-glycosidases and caspases in the cytotoxicity of novel glycoconjugates in colon cancer cells. Invest New Drugs 2010;28:306-17. doi:10.1007/s10637-0099248-2

24. Kolb HC, Finn MG, Sharpless KB. Click chemistry: Diverse chemical function from a few good reactions. Angew Chem Int Ed. 2011;40:2004-21. doi:10.1002/1521-3773(20010601)40:113.0.C0;25

25. Miller SJ. Cellular and physiological effects of short-chain fatty acids. Mini Rev Med Chem. 2004;4:839-45. doi:10.2174/1389557043403288

26. Tamura M, Okai H. Synthesis of 2-acetamido-1-N [N-(tert-butoxycarbonyl)-I-aspart-1-oyl-(Iphenylalanyl-I-serine methyl ester)-4-oyl]-2-deoxy- $\beta$-d-glucopyranosylamine and analogs. Carbohydr Res. 1984; 133:207-18. doi:10.1016/0008-6215(84)85199-X

27. Zi CT, Xu FQ, Li GT, Li Y, Ding ZT, Zhou J, et al. Synthesis and Anticancer Activity of Glucosylated Podophyllotoxin Derivatives Linked via 4ß-Triazole Rings. Molecules 2013;18:13992-4012. doi:10.3390/molecules181113992

28. Wang J, Sun PY, Wang Q, Zhang P, Wang YN, Zi CT, et al. (-)-Epigallocatechin-3-gallate derivatives combined with cisplatin exhibit synergistic inhibitory effects on non-small-cell lung cancer cells. Cancer Cell Int. 2019;19:1-16. doi:10.1186/s12935-019-0981-0

29. Jeong YC, Anwar M, Moloney MG. Synthesis, antibiotic activity and structure-activity relationship study of some 3-enaminetetramic acids. Bioorg Med Chem Lett. 2014;24:1190-200. doi:10.1016/j.bmcl.2014.03.013

\section{Tables}

Table 1 In vitro anticancer activity $\left(\mathrm{IC}_{50}, \mu \mathrm{M}\right)$ of triazole-linked gucose-(-)-epigallocatechin-3-gallate derivatives $10-13$. 


\begin{tabular}{|c|c|c|c|c|c|c|}
\hline \multirow[t]{2}{*}{ Compounds } & \multicolumn{6}{|c|}{$\mathrm{IC}_{50}(\mu \mathrm{M})$} \\
\hline & $\mathrm{HL}-60$ & SMMC-7721 & A-549 & MCF-7 & SW480 & BEAS-2B \\
\hline 10 & 4.56 & 23.32 & 38.48 & 36.47 & 38.21 & 38.66 \\
\hline 11 & 3.78 & 21.26 & 30.12 & 28.24 & 31.00 & 31.58 \\
\hline 12 & 13.47 & $>40$ & $>40$ & 35.60 & $>40$ & NT \\
\hline 13 & 15.30 & $>40$ & $>40$ & 39.89 & $>40$ & NT \\
\hline 4 & $>40$ & $>40$ & $>40$ & $>40$ & $>40$ & $>40$ \\
\hline cisplatin & 1.17 & 6.43 & 9.24 & 15.86 & 13.42 & 12.87 \\
\hline
\end{tabular}

Abbreviation: NT = not tested.

Table 2 The selectivity index of compounds 10,11 and cisplatin to cancer cells as compared with BEAS2B normal cell Line.

\begin{tabular}{|llllll|}
\hline Compounds & \multicolumn{5}{l}{ Selectivity index $\left(\mathrm{SI}^{\mathrm{a}}\right)$} \\
& HL-60 & SMMC-7721 & A-549 & MCF-7 & SW480 \\
\hline $\mathbf{1 0}$ & 8.4 & 1.7 & 1.0 & 1.1 & 1.0 \\
$\mathbf{1 1}$ & 8.4 & 1.5 & 1.0 & 1.1 & 1.0 \\
Cisplatin & 11.0 & 2.0 & 1.4 & 0.8 & 1.0 \\
\hline
\end{tabular}

Note: aSelectivity index $(\mathrm{SI})=\mathrm{IC}_{50}$ of the compound in BEAS-2B cell line/IC $\mathrm{C}_{50}$ of the compound in cancer cee line.

Table 3 The ClogP values and PBA of triazole-linked glucose-(-)-epigallocatechin-3-gallate derivatives 10 $-13$.

\begin{tabular}{|lllll|}
\hline Compounds & Molecular formula & m.p. $\left({ }^{\circ} \mathrm{C}\right)$ & Yield (\%) & ClogPa $^{\mathrm{a}}$ \\
\hline 10 & $\mathrm{C}_{47} \mathrm{H}_{55} \mathrm{~N}_{3} \mathrm{O}_{20}$ & $98-100$ & 80 & 6.84 \\
\hline 11 & $\mathrm{C}_{72} \mathrm{H}_{92} \mathrm{~N}_{6} \mathrm{O}_{29}$ & $92-93$ & 77 & 10.61 \\
\hline 12 & $\mathrm{C}_{31} \mathrm{H}_{31} \mathrm{~N}_{3} \mathrm{O}_{16}$ & $125-126$ & 55 & 0.50 \\
\hline 4 & $\mathrm{C}_{40} \mathrm{H}_{44} \mathrm{~N}_{6} \mathrm{O}_{21}$ & $120-121$ & 52 & -2.08 \\
\hline
\end{tabular}


${ }^{\mathrm{a}} \operatorname{Clog} \mathrm{P}=$ calculated partition coefficient.

Table 4 Solubilities of EGCG and its derivatives.

\begin{tabular}{|lll|}
\hline Compounds & Solubility in water ${ }^{\mathrm{a}}(\mathrm{mM})$ & Relative solubility \\
\hline 10 & $5.71 \pm 0.73$ & 0.3 \\
\hline 11 & $1.05 \pm 0.73$ & 0.06 \\
12 & $281.17 \pm 0.09$ & 17 \\
\hline 13 & $512.88 \pm 1.01$ & 31 \\
\hline 4 & $16.40 \pm 0.73$ & 1 \\
\hline
\end{tabular}

${ }^{\text {a }}$ Mean \pm standard deviation $(n=3)$.

\section{Figures}<smiles>Oc1cc(O)c2c(c1)O[C@H](c1ccc(O)c(O)c1)[C@H](O)C2</smiles>

(-)-Epicatechin $(\mathbf{E C}, \mathbf{1})$<smiles>Oc1cc(O)c2c(c1)O[C@H](c1cc(O)c(O)c(O)c1)[C@H](O)C2</smiles>

(-)-Epigallocatechin (EGC, 3)<smiles>O=C(O[C@H]1Cc2c(O)cc(O)cc2O[C@H]1c1ccc(O)c(O)c1)c1cc(O)c(O)c(O)c1</smiles>

(-)-Epicatechin-3-gallate (ECG, 2)<smiles>[R]c1cc([C@@H]2Oc3cc(O)cc(O)c3C[C@H]2OC(=O)c2c[Z7]c(O)c(O)c2[Z7])cc(O)c1O</smiles>

(-)-Epigallocatechin-3-gallate (EGCG, 4) 
Figure 1

Structures of (-)-epicatechin (EC, 1), (-)-epicatechin-3-gallate (ECG, 2), (-)-epigallocatechin (EGC, 3), (-)epigallocatechin-3-gallate (EGCG, 4).

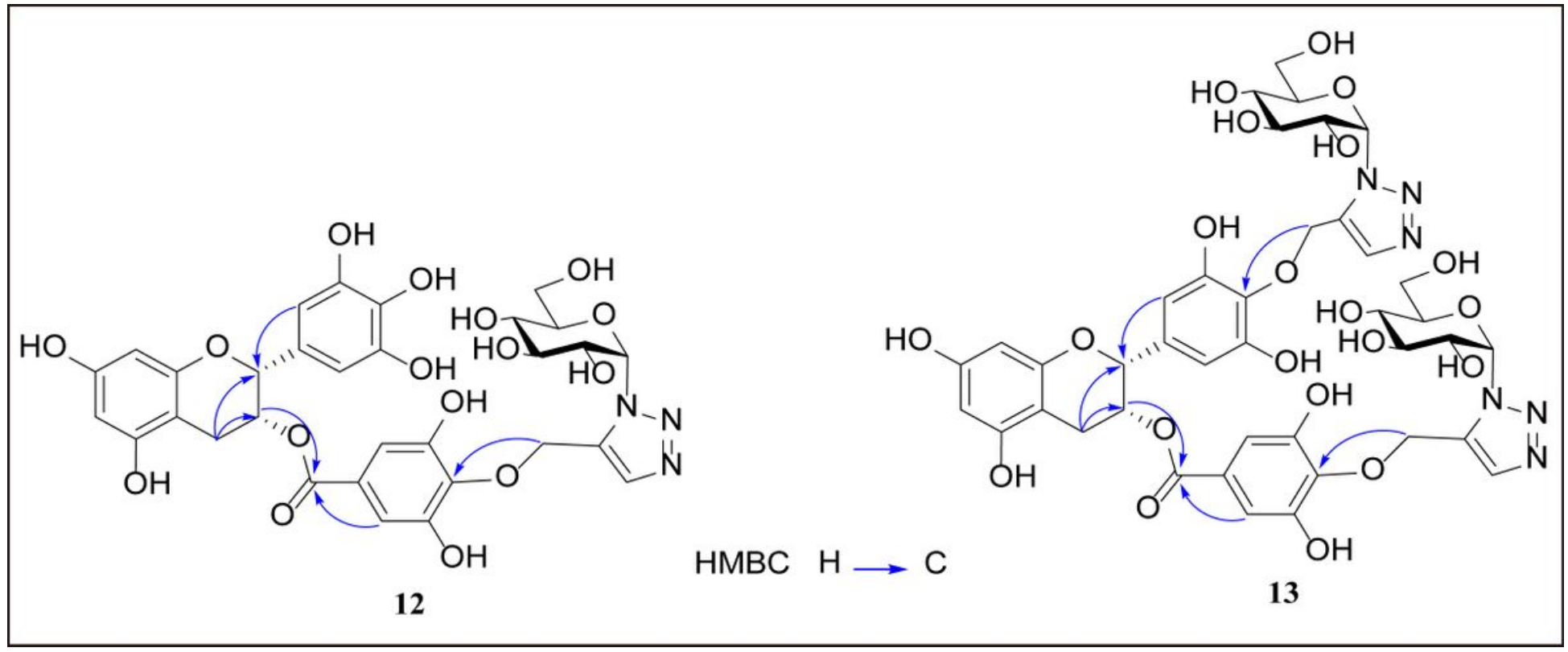

Figure 2

HMBC correlations in compounds 12 and 13.

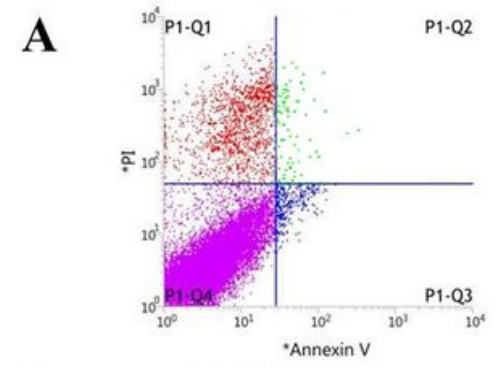

Compound $11(\mu \mathrm{M})$ -

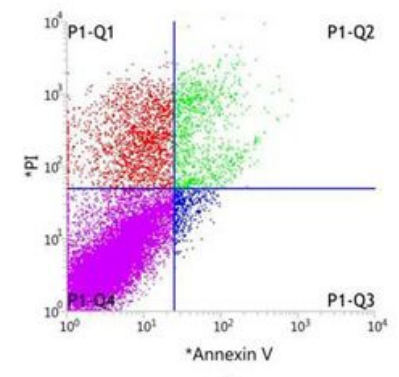

2

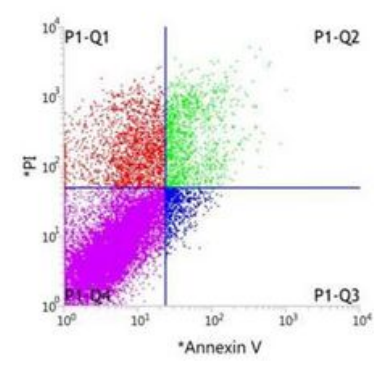

4

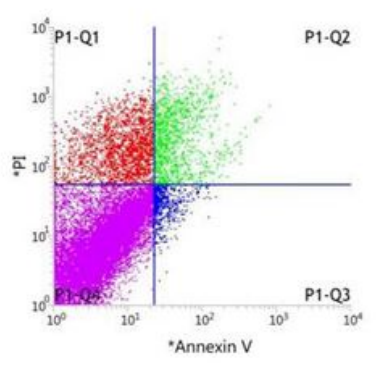

8
B

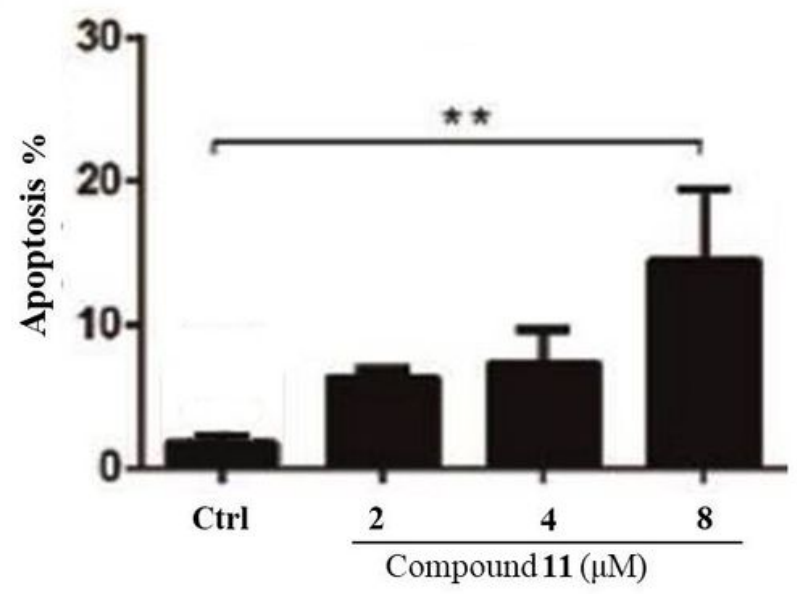

C

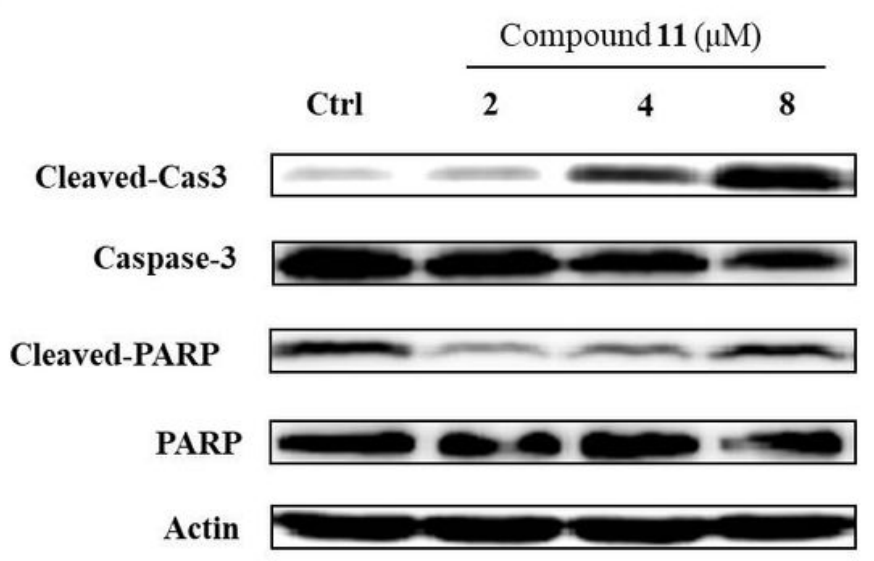

Figure 3 
Effect of compound 11 on the induction of cell apoptosis and the expression of relevant proteins in HL-60 cells.

\section{Supplementary Files}

This is a list of supplementary files associated with this preprint. Click to download.

- Scheme1.jpg 